\section{Between-subjects vs within-subjects comparisons of resistance to extinction}

\author{
ALLEN H. WOLACH, DOUGLAS HEGGI, and MORRIS ADERMAN \\ Mlinois Institute of Technology, Chicago, Ill. 60616
}

Two studies were performed to assess the effects of within-Ss and between-Ss $r$ sinforcement parameters on resistance to extinction. A two-way runway situation enabled rats to experience one sequence of reinforcements and nonreinforcements while running in one direction and another while running in the other direction (within-Ss comparison). Groups of Ss experienced different overall sequences (between-Ss comparisons). The two directional sequences determine 1 resistance to extinction when the nontransformed extinction data were examined. Manipulating the within-Ss reinforcement contingencies made it possible to obtain a "reverse" partial-reinforcement effect. Rate-corrected data (Anderson, 1963), however, were more sensitive to the overall sequence.

It is possible to generate many sequences with $\mathrm{N}$-lengths of 3 in one direction and N-lengths of 1,2 , and 3 in the other direction. These sequences produce overall N-lengths (independent of direction) that are different. Thus, even though two different groups of Ss experience $N$-lengths of 1,2 , and 3 in one direction and the N-length of 3 in the other direction, the maximum number of nonreinforced trials without regard to direction need not be the same for both groups. The Wolach, Coutts, \& Aderman (1970) study suggested that the two directional sequences, rather than the overall sequence, gained control over extinction performance. Extended training with an overall sequence that had a maximum N-length of 6 did not produce greater resistance to extinction than did an overall sequence that had a maximum N-length of 3 .

\section{EXPERIMENT 1}

The present study (Experiment 1) was designed to ascertain if the overall sequence of reinforcements can gain control (or partial control) over extinction performance. The two groups experienced the same sequence of nonreinforcements in both directions (N-length of 3 ). The first group experienced an overall $\mathrm{N}$-length of 1 , while the second group experienced an overall $\mathrm{N}$-length of 6 . If the two directional responses (within-Ss comparison) determine Ss' resistance to extinction, the overall $\mathrm{N}$-length without regard to direction (between-Ss comparison) should not be a critical determinant of resistance to extinction.

\section{Subjects}

Sixteen naive 120 - to 127-day-old male Sprague-Dawley rats served as Ss. The Ss were individually housed, with water available at all times. Two weeks prior to preliminary training, the Ss were placed on a 24-h feeding schedule. Three $2-\mathrm{cm}$ Noyes pellets were placed in each cage at the same time every day. During preliminary training and the experiment proper, the pellets were placed in a given cage $5 \mathrm{~min}$ after the $\mathrm{S}$ finished its daily session.

\section{Apparatus}

The apparatus was a straight alley runway, with a center section that was $193.0 \times 12.5 \times 14.5 \mathrm{~cm}$. Two guillotine-type doors separated the center section from two equal-sized end sections $(27.0 \times 12.4 \times 14.4 \mathrm{~cm})$. The walls of the runway were made of wood and painted flat black. The floor of the runway was constructed of steel tubing $(.1 \mathrm{~cm}$ thick $)$ that formed a grid with $1.0 \times 1.0 \mathrm{~cm}$ sections. The center and end sections of the runway were covered with removable framed sections of aluminum screening. Procedure

Two days of preliminary training preceded the experiment. The $S$ was placed in one end of the runway and allowed $10 \mathrm{~min}$ to traverse the runway. If $S$ did not reach the opposite end of the runway in $10 \mathrm{~min}$, it was gently pushed. The guillotine-type door closed behind the $S$ until it consumed two .045 -g Noyes pellets that were on a wooden tray at the back of the end section. The guillotine-type door was then raised and the next trial was initiated. All Ss experienced four preliminary training trials on both days of pretraining.

All Ss experienced a two-way runway situation during the experiment proper. A given $\mathbf{S}$ always

Table 1

Reinforcement Sequences for the Two Running Directions and the Combined Sequence Independent of Direction

\begin{tabular}{llll}
\hline & Direction 1 & Direction 2 & Overall Sequence \\
\hline $\begin{array}{l}\text { Group 1 } \\
\text { (Overall N- }\end{array}$ & NNNRRRRR & NNNRRRRR & NNNNNNRRRRRRRRRR \\
length 6) & NNNRRRRR & NNNRRRRR & NNNNNNRRRRRRRRRR \\
$\begin{array}{l}\text { Group 2 } \\
\text { (Overall N- }\end{array}$ & NNNRRRRR & RRRRNNNR & NRNRNRRRRNRNRNRR \\
length 1) & NNNRRRRR & RRRRNNNR & NRNRNRRRRNRNRNRR \\
\hline
\end{tabular}

started a daily session at the same end of the runway. When $S$ reached the opposite side of the runway, the guillotine-type door was closed behind it for $5 \mathrm{sec}$. Then the door was raised, and $S$ was required to run to the end section that had been the start section on the prior trial. The Ss experienced a total of 32 trials a day, 16 in each direction. Timing of the first trial was initiated when S's feet were placed on the starting-end section floor. The trial was terminated when $S$ was $16 \mathrm{~cm}$ into the opposite end compartment, and the guillotine-type door closed behind it. The remaining 31 trials were initiated by the raising of the guillotine-type door that confined $S$ in an end section. Any $S$ that failed to complete a trial in $90 \mathrm{sec}$ was gently pushed to the appropriate end of the runway and confined in the end section for 5 sec.

The Ss were assigned randomly to two equal groups, and each $S$ received 10 days of training. Table 1 shows the arrangement of the reinforced $(R)$ and nonreinforced (N) trials for each direction separately, as well as the overall sequence. A given $S$ experienced its sequence 10 times. It can be noted that $S$ s always experienced three nonreinforcements in a row ( $\mathrm{N}$-lengths of 3 ) in each direction. For instance, each $\mathbf{S}$ in the second group began with a nonreinforced trial (Direction 1), followed by a reinforced trial when it ran in the opposite direction (Direction 2), followed again by a nonreinforced trial when returning via Direction 1 (original direction), followed by another reinforced trial running in the second direction (Direction 2), and, finally, a third nonreinfored trial in Direction 1. The two groups differed in the number of nonreinforcements in a row (i.e., $\mathrm{N}$-length) independent of direction. Even though the two groups experienced $\mathrm{N}$-lengths of 3 in each direction, the number of nonreinforced trials, without regard to direction, was 1 for the first group and 6 for the second.

Four days of extinction followed the 10 days of acquisition. During extinction, the $\mathrm{Ss}$ experienced eight trials per day (four in each direction). 


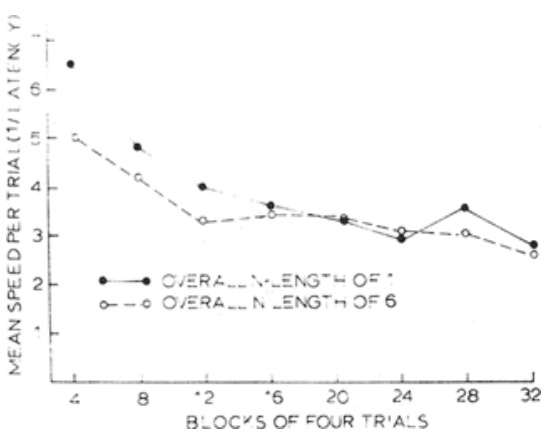

Fig. 1. Resistance to extinction for groups with overall N-lengths of 3 and 6.

\section{Results}

The data from the last half of the last day of acquisition training (i.e., the last 16 trials) were examined by means of a repeated measures analysis of variance (Running Direction by Experimental Group) to determine if the Ss' speeds were different in the two directions, or if the two groups (overall $\mathrm{N}$-length of 6 and overall $\mathrm{N}$-length of 1) differed. A given $S$ contributed 2 data points from the last day of acquisition. These data points consisted of the sum of the running speeds (1/latency in seconds) separately for the two directions over the last 16 trials of the session. Similar running speeds were found in both running directions $[F(1,14)=2.74$, $p>.05]$. The experimental group effect $[F(1,14)=.14, p>.05]$ and the Experimental Group by Running Direction interaction $[F(1,14)=2.79$, $p>.05$ ] were not significant. Consequently, the analysis of variance revealed that the running speeds in both directions and the overall running speeds were very similar at the end of acquisition.

The extinction data were analyzed to ascertain if the running speeds in the two directions or overall running speeds for the two groups differed during extinction. The data for each $\mathbf{S}$ consisted of the sum of running speeds (1/latency in seconds) in the two running directions across the 4 days of extinction. The two running directions did not produce different running speeds $[F(1,14)=.67, \mathrm{p}>.05]$. This was not surprising, since the $S$ received comparable reinforcement contingencies in the two directions. The experimental group effect (overall $\mathrm{N}$-length of 3 vs overall $\mathrm{N}$-length of 1) $[F(1,14)=.89, p>.05]$ and the Running Direction by Experimental Group interaction effect $[F(1,14)=1.02, \quad p>.05]$ were not significant. The running speeds for the two groups are presented in Fig. 1. Since the running speeds in the two directions did not differ, the data from the two directional responses are combined for each of the groups.

Between-group studies that employ extended training and $\mathrm{N}$-lengths as disparate as 1 and 6 consistently indicate greater resistance to extinction for the group with the longer N-length following extended training. The similar resistance to extinction in the present study for the groups with overall $\mathrm{N}$-lengths of 1 and 6 supports the contention that the two directional sequences (rather than the overall sequence) determine extinction behavior.

EXPERIMENT 2
It is obvious that a within-Ss design need not produce results that are similar to those obtained with between-Ss designs (see Grice \& Hunter, 1967). The within-Ss design provides the opportunity for Ss to form discriminations during acquisition that cannot be formed if Ss experience only one reinforcement condition. In addition, the opportunity for contrast effects can only occur if a within-Ss design is used. Experiment 2 is an attempt to examine a within-Ss partial reinforcement paradigm in which the conditions that have produced conventioanl between-Ss partial reinforcement effects (less resistance to extinction for groups with greater percentages of reinforcement during acquisition) are balanced against discrimination conditions during acquisition that could tend to mitigate a partial reinforcement effect. The Ss experienced running contingencies with one direction associated with $62.5 \%$ reinforcement and the other direction associated with $25 \%$ reinforcement. A further restriction on the directional sequences insured that the $62.5 \%$ reinforcement direction was always associated with $\mathrm{N}$-lengths of 1 , while the $25 \%$ reinforcement direction was consistently associated with $\mathrm{N}$-lengths of 6 . A prediction about resistance to extinction based on percentage of reinforcement or N-length (after extended training) would have to suggest greater resistance to extinction for the $62.5 \%$ reinforcement direction. As previously noted, within-Ss designs provide an opportunity for Ss to discriminate the two sequences during acquisition, whereas this kind of discrimination is not possible with a between-Ss design. Table 2

Reinforcement Sequences for the Two Running Directions and the Combined Sequence Independent of Direction

$$
\text { Direction } 1
$$

Direction 2 Overall Sequence

\section{Group 1}

Directional N-lengths of 6 and 1 , overall $\mathrm{N}$-length of 3

NNNNNNRR NNNNNNRR

NRNRNRRR NRNRNRRR

NNNRNNNRNNNRRRRR

Group 2

Directional N-lengths of 6 and 1 , overall $\mathrm{N}$-lengths of 3 and 1

\section{Method}

Sixteen naive 130- to 135-day-old male Sprague-Dawley rats served as Ss. The housing, apparatus, and preliminary training contingencies were the same as those used in Experiment 1. Table 2 illustrates the reinforcement sequences for the two groups. The training and extinction conditions (other than the reinforcement sequencing) were also the same as the ones used in Experiment 1.

A repeated measures analysis of variance (Running Direction by Experimental Group) showed the Ss directious $[F(1,14)=6539, p<01]$ during the last day of acquisition. The overall speed of the two experimental ups $[F(1,14)=.06, p>.05]$ and the Running Direction by Group interaction not significant.

The extinction data were analyzed o ascertain if running speeds in the directions differed during extinction. Figure 2 shows the running speeds for both groups in the two faster in the direction in which they experienced both the shorter $\mathrm{N}$-length and the greater percentage of einforcement $\quad[F(1,14)=54.60$, $p<.01]$. The two experimental groups (overall $\mathrm{N}$-length of 3 vs overal $\mathrm{N}$-lengths of 1 and 3 ) did not differ $[F(1,14)=.07, p>.05]$. The Group by Running irection interaction was also not cant $[F(1,14)=.00, p>.05]$

response rates for the $\mathrm{Ss}$ the end (Anderson, 1963) was performed, using each S's terminal acquisition data (i.e., the two directional responses were corrected separately). An estimate of a S's terminal acquisition speed (TA) in a given was obtained by averaging its ast four acquisition trials in that rection. The S's terminal extinction which is the reciprocal of $90 \mathrm{sec}$, the maximum time a $S$ was allowed per trial. The speed for each extinction trial was corrected with the following 


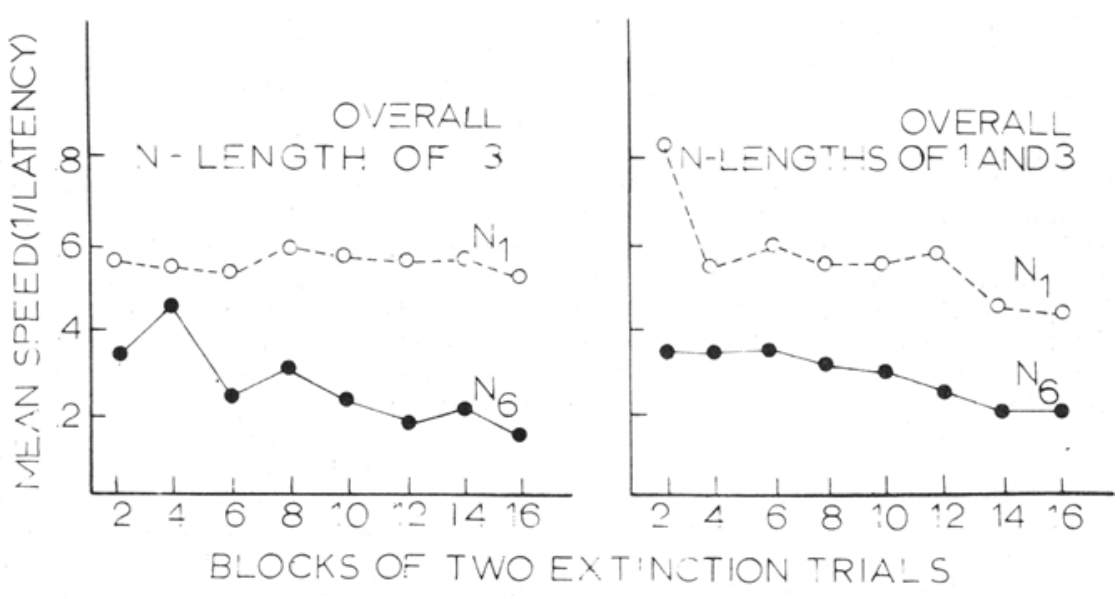

Fig. 2. Resistance to extinction in two running directions ( $\mathrm{N}$-length of $1 \mathrm{vs}$ $\mathrm{N}$-length of 6 ) for groups with overall N-lengths of 3 vs 1 and 3 .

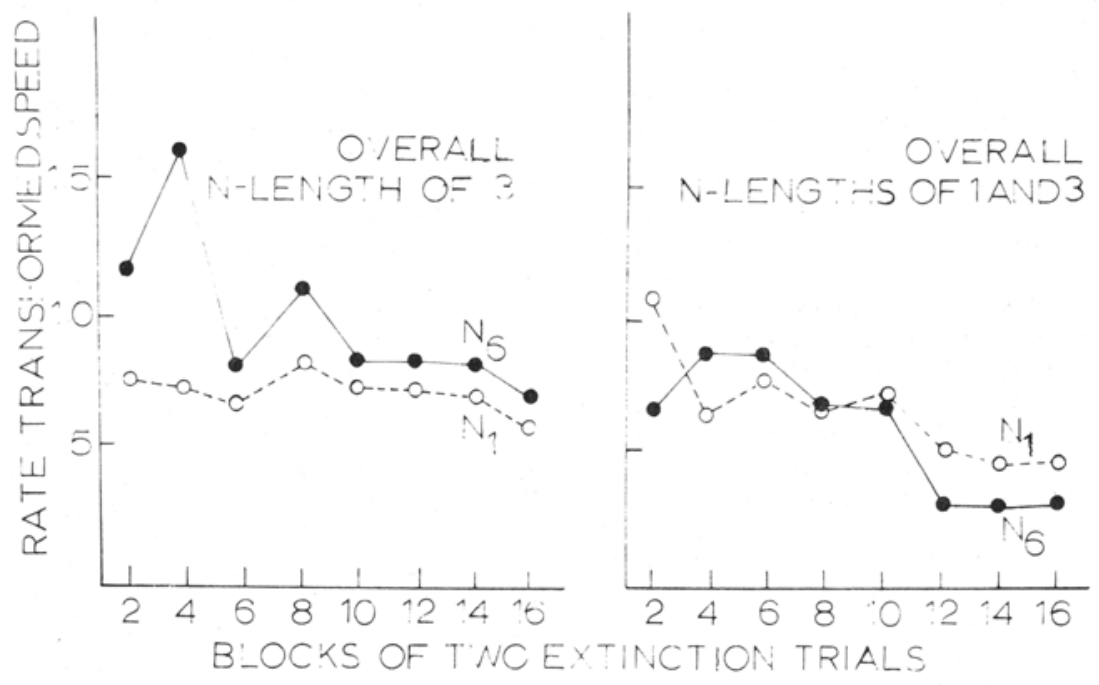

Fig. 3. Rate transformed resistance to extinction in two running directions (N-length of 1 vs N-length of 6 ) for groups with overall N-lengths of 3 vs 1 and 3 .

formula: rate transformed speed $=$ (extinction trial speed - TE)/ (TA - TE).

The individual S's transformed rates were averaged separately for the two directional responses and are plotted in Fig. 3. A repeated measures analysis of variance was also performed on the transformed data. The experimental group effect $[F(1,14)=6.67, p<.05]$ and the Experimental Group by Running Direction interaction effect $[F(1,14)=6.33, \quad p<.05]$ were significant. Running direction did not produce significant differences in running speed $[F(1,14)=1.47$, $\mathrm{p}>.05]$.

\section{DISCUSSION}

The nontransformed data from Experiment 2 provide additional evidence that the two directional responses, rather than the overall response independent of direction,
(Anderson, 1963) the data in an attempt to correct for the differing directional running speeds at the end of acquisition. The rate-transformed data suggest that the "reverse" partial reinforcement effect can be mainly attributed to the different speeds that the Ss had in the two directions at the end of acquisition. However, the rate transformation did not produce evidence for a conventional partial reinforcement effect, i.e., the rate transformation indicates similar resistance to extinction for the directions associated with N-lengths of 1 or 6. Thus, the within-Ss design is less likely to produce a partial reinforcement effect than the more frequently employed between-Ss design.

The rate-transformed data also indicate a significant Experimental Group by Running Direction interaction. This interaction suggests that a simple overall sequence ( $\mathrm{N}$-length of 3 ) is more likely to facilitate obtaining a partial reinforcement effect for the directional responses than a relatively more complicated overall sequence ( $\mathrm{N}$-lengths of 1 and 3 ). Although this finding appears reasonable, it is not obtained when one considers the nontransformed extinction data (Wolach, Coutts, \& Aderman, 1970). Similarly, only the transformed data suggest that the overall sequence (N-lengths of 3 vs 1 and 3 ) can gain any control over extinction performance. The rate-transformed data seem more sensitive to differences in the overall sequence than the nontransformed data. Conversely, the nontransformed data seem much more sensitive to the two directional sequences. The present study suggests that it might be useful to reexamine previous between-Ss partial reinforcement studies with rate transformations to observe how the transformation affects the data.

extinction performan The two directional responses yield evidence for what looks like a "reverse" partial reinforcement effect. Slower running speeds in extinction were obtained with the direction that was associated with the smaller percentage of reward and longer N-lengths. Although the longer $\mathrm{N}$-length produced a lower average running speed in extinction, the two $\mathrm{N}$-lengths did not produce different rates of extinction. This suggests that the "reverse" partial reinforcement effect is largely determined by the different directional running speeds that Ss develop by the end of acquisition. The within-Ss design and the discrimination that it afforded were apparently responsible for the large difference in running speeds at the end of acquisition. Consequently, it was decided to rate transform 